\title{
Editorial
}

\section{Management of Peritoneal Carcinomatosis: Is an Answer at Hand?}

\author{
Paul F. Mansfield, MD
}

In this issue of the Annals of Surgical Oncology, Glehen et al. ${ }^{1}$ report their results using intraperitoneal hyperthermic chemotherapy (IPCH) for the management of carcinomatosis in more than 200 patients. They report mortality and morbidity rates of $3.2 \%$ and $24.5 \%$, respectively. These favorable results join other studies in potentially supporting a more widespread use of IPCH. This publication raises an important and timely question. Whereas a number of us have accepted its use and perform this procedure routinely for appendiceal carcinoma with peritoneal metastases or disseminated peritoneal adenomucinosis, its precise role in other diseases has never been fully defined. Studies have reported a benefit in gastric cancer, whereas other studies have failed to find a benefit. Were IPCH a benign treatment, there would be less concern. Although it is easy to use prospective or even retrospective data for a rare disease (e.g., appendiceal neoplasm), with so few patients, it would be impossible for a randomized clinical trial to be completed during the lifetime of the investigators. The same is not true for common diseases.

In this study, patients with ovarian, colon, and gastric tumors predominated, with very few appendiceal neoplasms. Most importantly, this study looked at complications, not survival. Although it is critical to demonstrate that a treatment can be done with acceptable morbidity, reasoning that the treatment also seems to work (in selected patients) does not provide sufficient evidence for its widespread adoption to a disease such as colon cancer. The differences between appendiceal neo-

Received August 19, 2003; accepted August 22, 2003.

From the Department of Surgical Oncology, University of Texas M.D. Anderson Cancer Center, Houston, Texas.

Address correspondence to: Paul F. Mansfield, MD, Department of Surgical Oncology, University of Texas M.D. Anderson Cancer Center, 1515 Holcombe Blvd., Box 444, Houston, TX 77030; Fax: 713-7454926; E-mail: pmansfield@mdanderson.org.

Published by Lippincott Williams \& Wilkins $\odot 2003$ The Society of Surgical Oncology, Inc. plasms and colon cancer are dramatic. Hepatic and pulmonary metastases are common with colon cancer but rare in appendiceal neoplasms. Although the randomized trial presented by Zoetmulder ${ }^{2}$ at the American Society of Clinical Oncology last year demonstrated a survival advantage for patients having perfusion, significant methodologic concerns about this trial exist. Patients in the two arms of the study had different operations, the impact of which is unknown.

Although some may advocate for the adoption of perfusion as the standard of care for carcinomatosis from colon cancer, it is incumbent on us to perform the appropriate clinical trial when such a trial is feasible. Dr. H. Richard Alexander from the Nation Cancer Institute proposed such a trial at the recent American College of Surgical Oncology Group meeting in Montreal. ${ }^{3}$ With the study by Glehen ${ }^{1}$ and the report by Zoetmulder et al. ${ }^{2}$ in hand, it is imperative that we move forward with such a study. In designing such a study, it must be assured that the operations are the same, with the exception of the perfusion, thus requiring randomization only after completion of the debulking procedure. In addition, because of the multiple variables that can affect the outcome of patients with colon cancer, including degree of differentiation, molecular markers, and even the presence of other metastatic sites, these additional variables could be evaluated and stratified, when possible, or excluded. Given the large numbers of patients with colon cancer and the relative frequency of carcinomatosis, such a study could likely be completed in a timely fashion by the centers who currently perform perfusion in the United States. This study, however, would require an intense effort and commitment by those who perform this procedure. A significant critical mass of dedicated healthcare providers is necessary to safely care for these patients and the learning curve for their management is not insignificant. In addition, the cost of a perfusion is significant and we owe it to society and, most impor- 
tantly, to our patients to carefully evaluate this procedure. Is perfusion the answer? Maybe. Is it ready to be subjected to the scrutiny of a well-designed and conducted randomized trial? Absolutely.

\section{REFERENCES}

1. Glehen O, Osinsky D, Cotte E, et al. Intraperitoneal chemohyperthermia using a closed abdominal procedure and cytoreductive surgery for the treatment of peritoneal carcinomatosis: morbidity and mortality analysis of 216 consecutive procedures. Ann Surg Oncol 2003;10:863-9.
2. Zoetmulder FA, Verwaal VJ, Ruth S. Hyperthermic intra peritoneal chemotherapy (HIPEC) with mitomycin C significantly improves survival in patients with peritoneal carcinomatosis of colorectal origin. Paper presented at: Proceedings of the 38th Annual Meeting of the American Society of Clinical Oncology; May 19, 2002; Orlando, FL.

3. Alexander HR. A prospective random assignment trial for patients with peritoneal carcinomatosis from low-grade gastrointestinal adenocarcinoma to treatment with operative debulking and systematic chemotherapy with or without intra- and perioperative intraperitoneal chemotherapy. Paper presented at: American College of Surgeons Oncology Group; June 6, 2003 ; Montreal, Canada. 